\title{
PomCyDex - a lower-cost option in refractory myeloma?
}

PomCyDex...
might be a
reasonable
option
for many
patients with
lenalidomide-
refractory MM

Pomalidomide plus dexamethasone (PomDex) is a standard treatment for patients with multiple myeloma (MM) refractory to both proteasome inhibitors and lenalidomide. New findings suggest that a modified regimen including cyclophosphamide (PomCyDex) improves on the limited efficacy of PomDex.

In a randomized phase II study, 34 patients received PomCyDex and 36 received PomDex; the overall response rate was $64.7 \%$ versus $38.9 \%(P=0.0355)$, median progression-free survival was 9.5 months versus 4.4 months, and median overall survival was not reached versus 16.8 months. The survival differences were not statistically significant, but were noted despite crossover of $47 \%$ of the PomDex cohort to PomCyDex treatment upon disease progression. "Importantly, the addition of cyclophosphamide for patients with progressive disease on PomDex was associated with a very low response rate (6\%)," explains lead author Rachid Baz. "This experience suggests that PomCyDex is preferable, compared with PomDex followed by the addition of cyclophosphamide upon disease progression".

"A subgroup analysis revealed that patients without high-risk cytogenetics $(17 \mathrm{p}$ deletion or $\mathrm{t}(4 ; 14))$ seem to derive more benefit from PomCyDex than PomDex, whereas patients with high-risk cytogenetics seems to have better outcomes with PomDex," adds Baz. "This finding is thoughtprovoking, but tempered by the small sample size and unplanned nature of the analysis."

A number of drugs have been shown to increase the efficacy of PomDex; cyclophosphamide is a new addition to this list. "Importantly, as cyclophosphamide is off patent, PomCyDex is less costly than the other regimens that combine pomalidomide with proteasome inhibitors or monoclonal antibodies, and might be a reasonable option for many patients with lenalidomide-refractory MM," Baz concludes.

\section{David Killock}

ORIGINAL ARTICLE Baz, R. C. et al. Randomized multicenter phase II study of pomalidomide, cyclophosphamide, and dexamethasone in relapsed refractory myeloma. Blood http://dx.doi. org/10.1182/blood-2015-11-682518 (2016) 